Healthcare in Rural America: Annotated Questionnaire

October 2021 


\section{Screening Question}

D1. How old are you? CODES BELOW]

[RECORD NUMBER AND CREATE VARIABLE WITH THE

\begin{tabular}{|l|c|}
\hline & Total (\%) \\
\hline & 1,504 \\
\hline $40-44$ & 11 \\
\hline $45-49$ & 14 \\
\hline $50-59$ & 24 \\
\hline $60-69$ & 26 \\
\hline $70+$ & 25 \\
\hline Refused (terminate) & 0 \\
\hline
\end{tabular}

\section{Main}

General Health

Q1. How would you rate your overall health? Would you say it is excellent, very good, good, fair, or poor?

\begin{tabular}{|l|c|}
\hline & $\begin{array}{c}\text { Total } \\
(\%)\end{array}$ \\
\hline Excellent & 1,504 \\
\hline Very good & 13 \\
\hline Good & 29 \\
\hline Fair & 32 \\
\hline Poor & 17 \\
\hline Don't know/refused & 9 \\
\hline
\end{tabular}

Q2. Over the past two years, would you say your overall health has gotten better, stayed the same, or gotten worse?

\begin{tabular}{|l|c|}
\hline & $\begin{array}{c}\text { Total } \\
\text { (\%) }\end{array}$ \\
\hline & 1,504 \\
\hline Gotten better & 12 \\
\hline Stayed same & 61 \\
\hline Gotten worse & 27 \\
\hline Don't know/refused & $<1$ \\
\hline
\end{tabular}


Q3. How would you describe your mental health at this point in time? Would you say it is excellent, very good, good, fair, or poor?

\begin{tabular}{|l|c|}
\hline & $\begin{array}{c}\text { Total } \\
(\%)\end{array}$ \\
\hline & 1,504 \\
\hline Excellent & 26 \\
\hline Very good & 33 \\
\hline Good & 26 \\
\hline Fair & 12 \\
\hline Poor & 3 \\
\hline Don't know/refused & $<1$ \\
\hline
\end{tabular}

Q4. Many people have various health conditions. Please tell me if you have any of the following health conditions. [Please select all that apply.] (Randomize list) [Interviewer note: Do not pressure respondent to answer this question if they do not want to - simply mark "Refused" and move on]

\begin{tabular}{|l|c|}
\hline & $\begin{array}{c}\text { Total } \\
(\%)\end{array}$ \\
\hline & 1,504 \\
\hline High cholesterol & 30 \\
\hline High blood pressure & 43 \\
\hline Heart disease & 15 \\
\hline Diabetes & 22 \\
\hline Gastric distress & 11 \\
\hline Asthma & 9 \\
\hline Respiratory disease (such as lung disease, COPD, emphysema) & 11 \\
\hline Depression & 18 \\
\hline Anxiety & 22 \\
\hline Cancer & 7 \\
\hline Chronic pain & 27 \\
\hline Complications due to a fall, broken bone or other physical injury & 14 \\
\hline Arthritis & 41 \\
\hline Osteoporosis & 9 \\
\hline Alzheimer's or dementia & 2 \\
\hline I do not have any of these health conditions (Anchor, exclusive) (do not & 20 \\
\hline read) & \\
\hline Don't know/refused (do not read) & 1 \\
\hline
\end{tabular}




\section{AARP}

Healthcare Access, Quality, and Costs

Q5. Where do you usually go for treatment when you are sick with a non-life-threatening illness? Would you say you usually go to...? (Randomize list) (Read list; accept one response)

\begin{tabular}{|l|c|}
\hline & $\begin{array}{c}\text { Total } \\
(\%)\end{array}$ \\
\hline & 1,504 \\
\hline A doctor's office & 61 \\
\hline Hospital emergency room & 4 \\
\hline Urgent care facility (not at a hospital) & 4 \\
\hline Private clinic or health center & 10 \\
\hline Public clinic or health center & 9 \\
\hline VA / Military hospital or clinic & 4 \\
\hline Hospital outpatient department & 3 \\
\hline Other & 2 \\
\hline I don't seek treatment when I am sick (Anchor, Exclusive) (do not read) & 3 \\
\hline Don't know/refused (do not read) & 1 \\
\hline
\end{tabular}

Q6. Do you have a regular doctor or health care provider you go to when you are sick or want medical advice?

\begin{tabular}{|l|c|}
\hline & $\begin{array}{c}\text { Total } \\
(\%)\end{array}$ \\
\hline & 1,504 \\
\hline Yes & 89 \\
\hline No & 11 \\
\hline Don't know/refused & $<1$ \\
\hline
\end{tabular}

Q6A. [If yes to Q6] When was the last time you went to your regular doctor or health care provider? (Read list; accept one response)

\begin{tabular}{|l|c|}
\hline & $\begin{array}{c}\text { Total } \\
(\%)\end{array}$ \\
\hline & 1,339 \\
\hline Less than 3 months ago & 55 \\
\hline 3 to less than 6 months ago & 22 \\
\hline 6 months to a year ago & 15 \\
\hline Between 1 to 2 years ago & 6 \\
\hline More than 2 years ago & 3 \\
\hline Don't know/refused (do not read) & $<1$ \\
\hline
\end{tabular}


Q6B. [If no to Q6] What do you do when you need medical advice or attention? (Randomize list) (Read list; accept multiple responses)

\begin{tabular}{|l|c|}
\hline & $\begin{array}{c}\text { Total } \\
\text { (\%) }\end{array}$ \\
\hline & 163 \\
\hline You try to solve the problem on your own & 65 \\
\hline You go to the emergency room or medical clinic & 43 \\
\hline You go to the pharmacy / pharmacist & 19 \\
\hline You go online / on the Internet for information or advice & 34 \\
\hline You ask family / friends for information or advice & 35 \\
\hline None of the above (anchor, mutually exclusive) (do not read) & 5 \\
\hline Don't know/refused (do not read) & 1 \\
\hline
\end{tabular}

Q6C. [If no to Q6] When was the last time you saw a doctor, health care provider or medical facility to receive medical care? (Read list; accept one response)

\begin{tabular}{|l|c|}
\hline & $\begin{array}{c}\text { Total } \\
(\%)\end{array}$ \\
\hline & 163 \\
\hline Less than 3 months ago & 22 \\
\hline 3 to less than 6 months ago & 9 \\
\hline 6 months to a year ago & 10 \\
\hline Between 1 to 2 years ago & 16 \\
\hline More than 2 years ago & 42 \\
\hline Don't know/refused & 2 \\
\hline
\end{tabular}

Q7. [If yes to Q6] What was the main reason for your last visit for medical care? (Randomize) (Read list; accept one response)

\begin{tabular}{|l|c|}
\hline & $\begin{array}{c}\text { Total } \\
\text { (\%) }\end{array}$ \\
\hline & 1,339 \\
\hline For a regular check-up & 55 \\
\hline To get a flu or pneumonia shot & 6 \\
\hline To get a few medical tests & 3 \\
\hline For blood work & 11 \\
\hline For a health emergency & 3 \\
\hline For some other reason (anchor) & 22 \\
\hline Don't know/refused & 1 \\
\hline
\end{tabular}


Q8. At your most recent medical appointment, which of the following topics-if any-did a doctor or healthcare professional discuss with you? (Randomize list) (Select all that apply)

\begin{tabular}{|l|c|}
\hline & $\begin{array}{c}\text { Total } \\
\text { (\%) }\end{array}$ \\
\hline & 1,504 \\
\hline Diet and weight & 32 \\
\hline Exercise & 37 \\
\hline Depression or anxiety & 20 \\
\hline Alcohol use & 10 \\
\hline Smoking or tobacco use & 18 \\
\hline Sexual health & 8 \\
\hline Taking aspirin daily & 21 \\
\hline Ways to lower your prescription drug costs & 6 \\
\hline Ways to maintain your brain health, stay cognitively sharp, and avoid & 9 \\
\hline dementia & 16 \\
\hline Taking dietary or nutritional supplements & 42 \\
\hline Immunizations (such as the flu shot or pneumonia shot) & 6 \\
\hline Illegal drug use & 14 \\
\hline Eye health & 10 \\
\hline Dental health & 23 \\
\hline None of these [Anchor, exclusive] (do not read) & 1 \\
\hline Don't know/refused (do not read) & \\
\hline
\end{tabular}

Q9/Q10. In the last 2 years, has there been a time when you needed medical care but you...? (1=Yes, 2=No, 9=Don't know/refused)

\begin{tabular}{|c|c|}
\hline & $\begin{array}{c}\text { Total } \\
(\%)\end{array}$ \\
\hline & 1,504 \\
\hline & \\
\hline Delayed it? & 28 \\
\hline Did not get it? & 13 \\
\hline
\end{tabular}




\section{AARP}

Q11. [Among those who delayed or did not get medical care in Q9 or Q10] Why did you (if $\mathrm{Q} 9=1$ : delay getting; if $\mathrm{Q} 10=1$ : not get; if $\mathrm{Q9}=1$ and $\mathrm{Q} 10=1$ : delay getting or not get) the medical care you needed? (Randomize) (Read list; select all that apply.)

\begin{tabular}{|l|c|}
\hline & $\begin{array}{c}\text { Total } \\
\text { (\%) }\end{array}$ \\
\hline & 445 \\
\hline You couldn't find a doctor & 5 \\
\hline You didn't have time & 16 \\
\hline You thought it costs too much & 22 \\
\hline You didn't have health insurance & 9 \\
\hline You couldn't get an appointment & 12 \\
\hline It's too far to get there & 6 \\
\hline You didn't have a way to get there & 6 \\
\hline You couldn't take time off from work & 9 \\
\hline You didn't have anyone to care for your children or other family members & 4 \\
\hline You were concerned about social distancing or staying safe during the & 27 \\
\hline pandemic & \\
\hline A reason not listed (do not read, exclusive) & 30 \\
\hline Don't know/refused (do not read) & 2 \\
\hline
\end{tabular}

Q12. Within the past two years, have you or anyone in your family had a problem paying for your medical bills? Was that a major problem or minor problem for you?

\begin{tabular}{|l|c|}
\hline & $\begin{array}{c}\text { Total } \\
\text { (\%) }\end{array}$ \\
\hline & 1,504 \\
\hline Yes, major problem & 10 \\
\hline Yes, minor problem & 7 \\
\hline No & 83 \\
\hline Don't know/refused & $<1$ \\
\hline
\end{tabular}


AARP'

Oral/Dental Health Care

Q13. About how long has it been since you last saw a dentist or dental hygienist for dental care?

\begin{tabular}{|l|c|}
\hline & $\begin{array}{c}\text { Total } \\
(\%)\end{array}$ \\
\hline & 1,504 \\
\hline Less than one year & 54 \\
\hline One to two years & 20 \\
\hline Three to five years & 9 \\
\hline More than five years & 15 \\
\hline Never & 1 \\
\hline Don't know/refused & 1 \\
\hline
\end{tabular}

Q14. [If Q13=1-4] What was the main reason for your last visit for dental care? (Randomize) (Read list; accept one response)

\begin{tabular}{|l|c|}
\hline & $\begin{array}{c}\text { Total } \\
(\%)\end{array}$ \\
\hline & 1,473 \\
\hline Regular check-up & 26 \\
\hline For teeth cleaning & 31 \\
\hline To have a tooth or teeth filled or pulled & 18 \\
\hline Trouble with your gums & 1 \\
\hline A tooth ache & 2 \\
\hline Preparation, adjustment, or repair of full or partial dentures & 13 \\
\hline For a dental emergency & 2 \\
\hline For some other reason (anchor) & 6 \\
\hline Don't know/refused & 1 \\
\hline
\end{tabular}

Q15./Q16. In the last 2 years, has there been a time when you needed dental care but you...? (1=Yes, 2=No, 9=Don't know/refused)

\begin{tabular}{|l|c|}
\hline & $\begin{array}{c}\text { Total } \\
(\%)\end{array}$ \\
\hline Delayed dental care? & 1,504 \\
\hline Did not get dental care? & 26 \\
\hline
\end{tabular}




\section{AARP}

Q17. [Among those who delayed or did not get dental care] Why did you (if Q15=1: delay getting; if Q16=1: not get; if Q15=1 and Q16=1: delay getting or not get) the dental care you needed? (Randomize) (Read list; select all that apply.)

\begin{tabular}{|c|c|} 
& $\begin{array}{c}\text { Total } \\
(\%)\end{array}$ \\
\hline & 422 \\
\hline You couldn't find a dentist & 6 \\
\hline You were waiting until you had dental health insurance & 12 \\
\hline You didn't have time & 9 \\
\hline You thought it costs too much & 44 \\
\hline You don't have dental insurance & 26 \\
\hline You couldn't get an appointment & 8 \\
\hline It's too far to get there & 4 \\
\hline You didn't have a way to get there & 4 \\
\hline You couldn't take time off from work & 5 \\
\hline You didn't have anyone to care for your children or other family members & 2 \\
\hline You were concerned about social distancing or staying safe during the & 23 \\
\hline pandemic & \\
\hline A reason not listed (do not read, exclusive) & 17 \\
\hline Don't know/refused (do not read) & $<1$ \\
\hline
\end{tabular}

Q18. Have you or anyone in your family ever had a problem paying for your dental bills? Was that a major problem or minor problem for you?

\begin{tabular}{|l|c|}
\hline & $\begin{array}{c}\text { Total } \\
(\%)\end{array}$ \\
\hline & 1,504 \\
\hline Yes, major problem & 11 \\
\hline Yes, minor problem & 7 \\
\hline No & 82 \\
\hline Don't know/refused & 1 \\
\hline
\end{tabular}

\section{Healthcare Quality}

Q20. Was there ever a time in the past two years when you felt there was a problem with the quality of healthcare you received?

\begin{tabular}{|l|c|}
\hline & $\begin{array}{c}\text { Total } \\
(\%)\end{array}$ \\
\hline & 1,504 \\
\hline Yes & 15 \\
\hline No & 84 \\
\hline Don't know/refused & 1 \\
\hline
\end{tabular}




\section{AARP}

Q21. Have any hospitals or medical clinics in your local community recently closed down?

\begin{tabular}{|l|c|}
\hline & $\begin{array}{c}\text { Total } \\
(\%)\end{array}$ \\
\hline & 1,504 \\
\hline Yes & 10 \\
\hline No & 84 \\
\hline Don't know/refused & 5 \\
\hline
\end{tabular}

Q22. Do you have access to the following health care services in your community? (Randomize)

\begin{tabular}{|c|c|}
\hline & $\begin{array}{c}\text { Total } \\
(\%)\end{array}$ \\
\hline & 1,504 \\
\hline Percentage Yes & \\
\hline Dental services & 90 \\
\hline Walk-in ("Minute") clinics & 76 \\
\hline Pharmacies & 96 \\
\hline Home health care services & 83 \\
\hline Hospice or palliative care services & 79 \\
\hline Mental health services & 72 \\
\hline Substance abuse services & 64 \\
\hline Obstetrics and other women's health services & 69 \\
\hline $\begin{array}{l}\text { Specialty care services (such as a cardiologist, neurologist, dermatologist, } \\
\text { or other specialist) }\end{array}$ & 74 \\
\hline
\end{tabular}

Q23. Because of a physical, mental, or emotional condition, do you need the help of other people...? [INSERT AND READ IN ORDER] (Select all that apply)

\begin{tabular}{|l|c|}
\hline & $\begin{array}{c}\text { Total } \\
(\%)\end{array}$ \\
\hline & 1,504 \\
\hline $\begin{array}{l}\text { With personal care needs, such as eating, bathing, dressing, or getting } \\
\text { around inside your home? }\end{array}$ & 6 \\
\hline $\begin{array}{l}\text { To get around outside your home, such as running errands, shopping, or } \\
\text { visiting a doctor's office? }\end{array}$ & 13 \\
\hline To manage your finances? & 6 \\
\hline To manage any medications or medical treatments? & 6 \\
\hline With household chores, such as cleaning or laundry? & 80 \\
\hline None of the above (do not read; exclusive) & 82 \\
\hline
\end{tabular}




\section{AARP}

Q24A. [If Q23 = yes for any] How familiar, if at all, would you say you are with organizations or groups in your community that provide information or assistance in helping you to find the support you need?

\begin{tabular}{|l|c|}
\hline & $\begin{array}{c}\text { Total } \\
\text { (\%) }\end{array}$ \\
\hline & 299 \\
\hline Extremely familiar & 12 \\
\hline Very familiar & 22 \\
\hline Somewhat familiar & 24 \\
\hline Not very familiar & 15 \\
\hline Not at all familiar & 23 \\
\hline Don't know/refused & 5 \\
\hline
\end{tabular}

Q24B. [If 23 = none of the above] How familiar, if at all, would you say you are with organizations or groups in your community that provide information or assistance to individuals who need support with those activities? (Read list)

\begin{tabular}{|l|c|}
\hline & $\begin{array}{c}\text { Total } \\
(\%)\end{array}$ \\
\hline & 1,205 \\
\hline Extremely familiar & 9 \\
\hline Very familiar & 21 \\
\hline Somewhat familiar & 35 \\
\hline Not very familiar & 13 \\
\hline Not at all familiar & 21 \\
\hline Don't know/refused & 2 \\
\hline
\end{tabular}

\section{Internet Use}

Q25. Do you have broadband access in your community? [Definition if needed: The term broadband commonly refers to high-speed Internet access that is always on and faster than the traditional dial-up access.]

\begin{tabular}{|l|c|}
\hline & $\begin{array}{c}\text { Total } \\
\text { (\%) }\end{array}$ \\
\hline & 1,504 \\
\hline Yes & 77 \\
\hline No & 16 \\
\hline Don't know/refused & 7 \\
\hline
\end{tabular}




\section{AARP}

Q26. Which of the following items do you have or own? [RANDOMIZE] (Read list; select all that apply)

\begin{tabular}{|l|c|}
\hline & $\begin{array}{c}\text { Total } \\
(\%)\end{array}$ \\
\hline & 1,504 \\
\hline Desktop computer & 47 \\
\hline Laptop computer or Netbook & 57 \\
\hline Smartphone such as iPhone, Android, Blackberry & 64 \\
\hline Regular cell phone (not including iPhone or other smartphones) & 44 \\
\hline E-reader such as Kindle or Nook & 23 \\
\hline Tablet such as iPad & 47 \\
\hline Wearable device such as Fitbit, Garmin, Apple Watch, Samsung Galaxy & 19 \\
\hline Watch & 19 \\
\hline Home assistant such as Amazon Echo or Alexa, Google Home & 15 \\
\hline $\begin{array}{l}\text { Smart home technology/security such as a smart thermostat, Nest, Ring, } \\
\text { SimpliSafe }\end{array}$ & 11 \\
\hline $\begin{array}{l}\text { GPS tracker specifically designed to monitor and share location such as a } \\
\text { necklace, bracelet, or watch }\end{array}$ & 4 \\
\hline Virtual reality (VR) device & 6 \\
\hline None of the above [ANCHOR] (Do not read, exclusive) & $<1$ \\
\hline Don't know/refused & \\
\hline
\end{tabular}

Q27. How often do you use the Internet? This includes using email and accessing the Internet through devices such as computers, tablets, or smartphones. (Read list)

\begin{tabular}{|l|c|}
\hline & $\begin{array}{c}\text { Total } \\
\text { (\%) }\end{array}$ \\
\hline & 1,504 \\
\hline More than once a day & 54 \\
\hline Once a day & 12 \\
\hline A few times a week & 8 \\
\hline A few times a month & 4 \\
\hline A few times a year or less & 3 \\
\hline Never & 18 \\
\hline Don't know/refused (Do not read) & 1 \\
\hline
\end{tabular}




\section{AARP}

Q28. [If Q27=1-5] Now, l'd like you to think about all of the places in your community in which access to the Internet is available to you, even if you haven't actually used the Internet at these places. In which of the following places is access to the Internet available to you? [READ LIST] [MULTIPLE RESPONSES ALLOWED] (Randomize)

\begin{tabular}{|l|c|}
\hline & $\begin{array}{c}\text { Total } \\
\text { (\%) }\end{array}$ \\
\hline & 1,221 \\
\hline At home & 90 \\
\hline At work & 48 \\
\hline At a library or school & 84 \\
\hline At a community center or senior center & 38 \\
\hline At a place of worship & 36 \\
\hline At a coffee shop or business other than your workplace & 61 \\
\hline Some other place & 28 \\
\hline None of the above (no access) (do not read; exclusive) & 1 \\
\hline Don't know/refused (do not read) & 1 \\
\hline
\end{tabular}

Q29. [If Q28=1] In the past 12 months, to what extent has each of the following limited your use of the Internet at home? To a great extent, to some extent, not too much, not at all? (Randomize)

\begin{tabular}{|l|r|r|}
\hline & & \multicolumn{1}{|c|}{$\begin{array}{c}\text { Total } \\
\text { (\%) }\end{array}$} \\
\hline & & 1,100 \\
\hline Cost of Internet access & To a great extent & 7 \\
\hline & To some extent & 14 \\
\hline & Not too much & 12 \\
\hline & Not at all & 64 \\
\hline Quality of Internet access & & \\
\hline & To a great extent & 12 \\
\hline & To some extent & 20 \\
\hline & Not too much & 19 \\
\hline Availability of Internet access & Not at all & 48 \\
\hline & & \\
\hline & To a great extent & 8 \\
\hline & To some extent & 14 \\
\hline & Not too much & 16 \\
\hline
\end{tabular}


Q30. [If Q28=1] What type of Internet connections do you have at home? (Randomize) (Read list; select all that apply)

\begin{tabular}{|l|c|}
\hline & $\begin{array}{c}\text { Total } \\
(\%)\end{array}$ \\
\hline & 1,100 \\
\hline Dial-up telephone service & 13 \\
\hline Broadband high-speed service such as cable, fiber-optic, DSL connection & 74 \\
\hline Satellite Internet connection & 19 \\
\hline Cellular data plan for a smartphone or other mobile device (anchor) & 46 \\
\hline Some other type of Internet connection (anchor) & 7 \\
\hline Don't know/refused (do not read) & 3 \\
\hline
\end{tabular}

Telehealth Knowledge and Use

Q31. About how long would it take you, in minutes, to get to...? (Randomize)

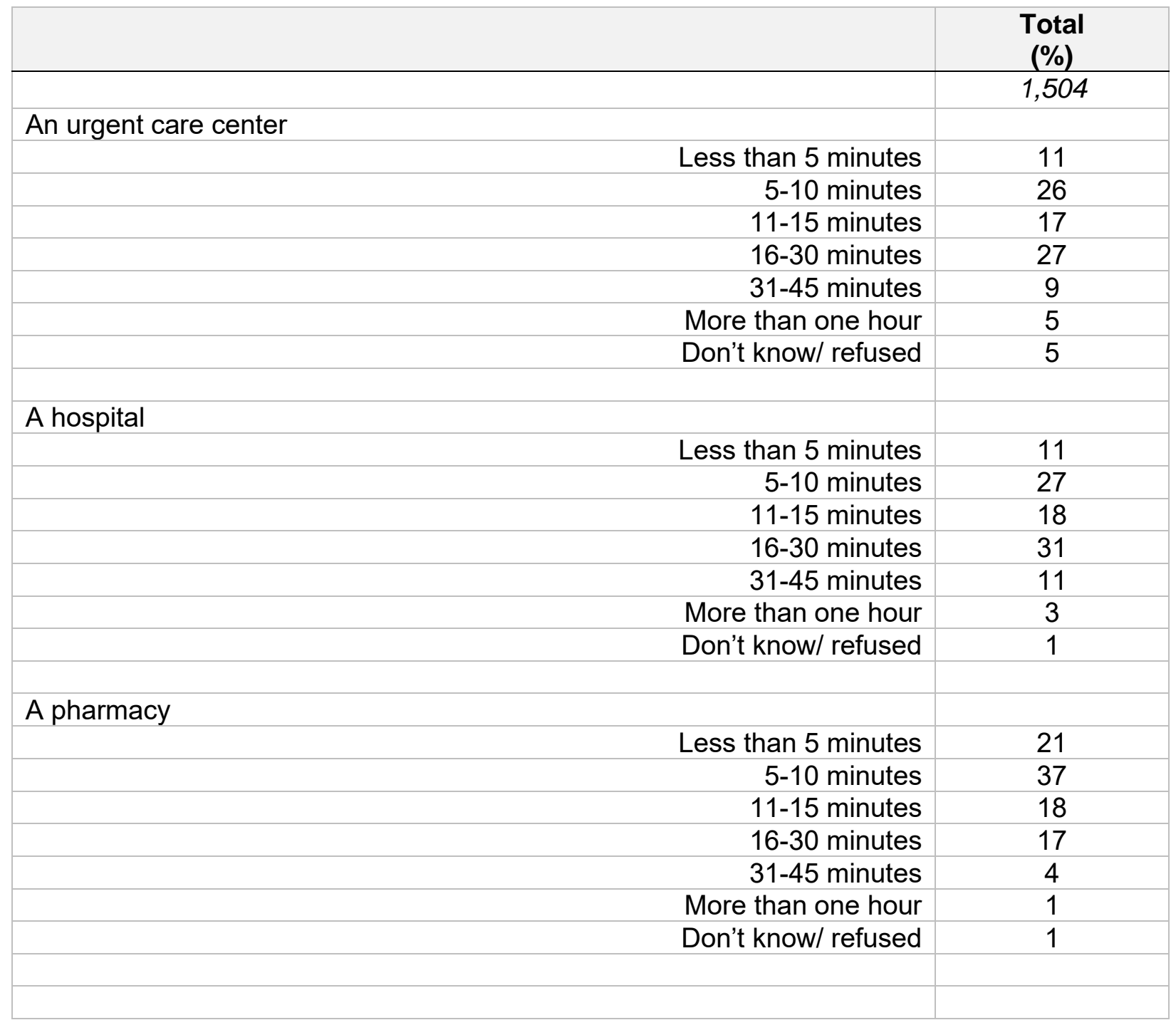


AARP'

A doctor's office for primary care

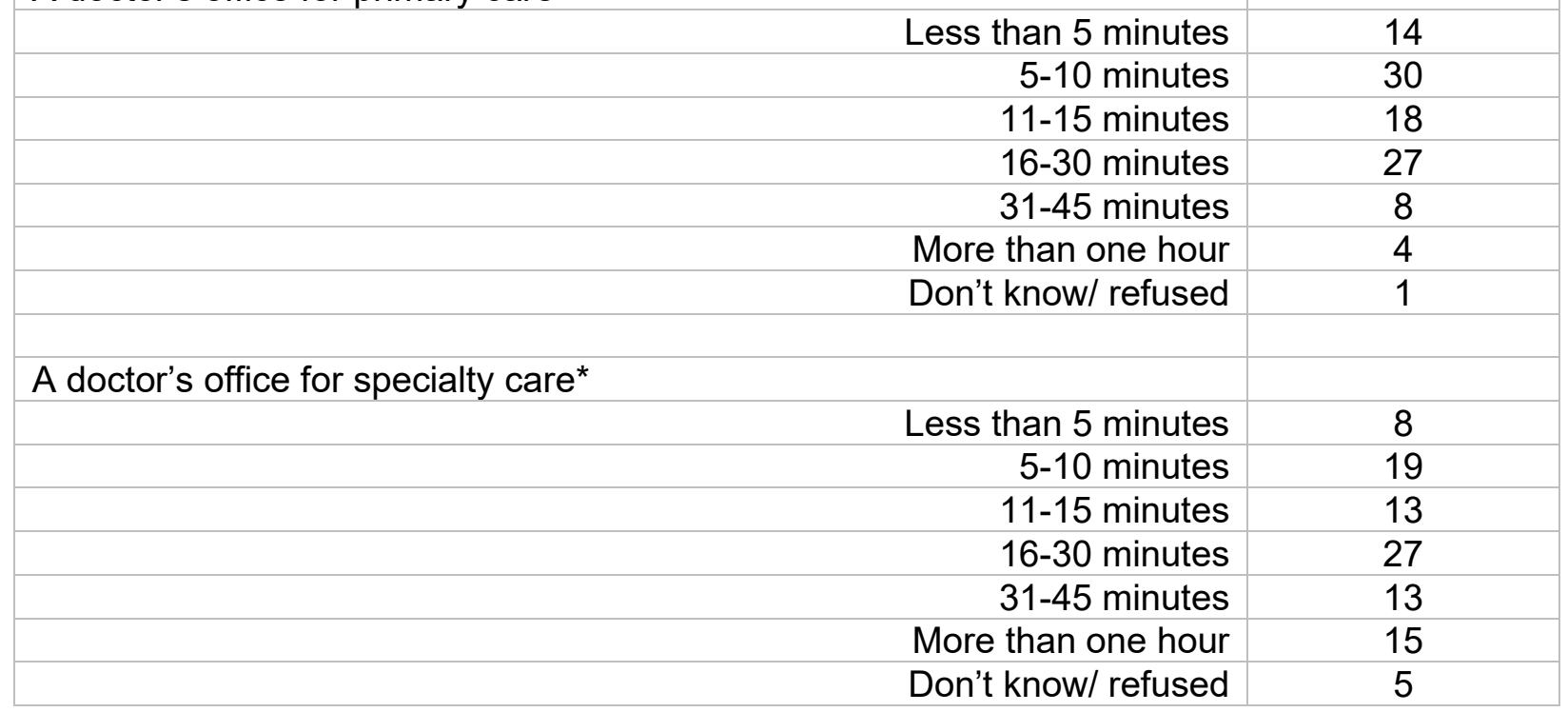

*if needed: like a cardiologist, neurologist, dermatologist, or other specialist

Q32. About how long do you think you it would take for you to get an appointment for a primary care visit? (Read list; accept one response)

\begin{tabular}{|l|c|}
\hline & $\begin{array}{c}\text { Total } \\
(\%)\end{array}$ \\
\hline & 1,504 \\
\hline Within a few days & 60 \\
\hline Between one and two weeks & 22 \\
\hline Between two weeks and one month & 8 \\
\hline Between one and three months & 4 \\
\hline More than three months & 1 \\
\hline Don't know/refused (do not read) & 5 \\
\hline
\end{tabular}

Q33. And about how long do you think it would take for you to get an appointment with a medical specialist? (Read list; accept one response)

\begin{tabular}{|l|c|}
\hline & $\begin{array}{c}\text { Total } \\
(\%)\end{array}$ \\
\hline & 1,504 \\
\hline Within a few days & 18 \\
\hline Between one and two weeks & 28 \\
\hline Between two weeks and one month & 24 \\
\hline Between one and three months & 16 \\
\hline More than three months & 4 \\
\hline Don't know/refused (do not read) & 11 \\
\hline
\end{tabular}




\section{AARP}

Q34. Have you heard of the following terms? (Randomize) (1=Yes, 2=No, 9=Don't know/refused)

\begin{tabular}{|c|c|}
\hline & $\begin{array}{c}\text { Total } \\
(\%)\end{array}$ \\
\hline & 1,504 \\
\hline & \\
\hline Telemedicine & 52 \\
\hline Telehealth & 56 \\
\hline Teledoc & 42 \\
\hline
\end{tabular}

Q35. Telehealth is another way for people to have one-on-one interactions with their health care providers without having to be in the same location at the same time by using cell phones, computers, or tablets. Examples of telehealth include a patient wearing a monitoring device that tracks vital signs or alerts a family caregiver if there is a serious injury, or an online conversation between patients and their doctors to get a diagnosis, a prescription filled, or a second opinion.

How interested are you in using telehealth services for yourself or a loved one if needed? Are you ...? (Read list)

\begin{tabular}{|l|c|}
\hline & $\begin{array}{c}\text { Total } \\
\text { (\%) }\end{array}$ \\
\hline & 1,504 \\
\hline Extremely interested & 5 \\
\hline Very interested & 7 \\
\hline Somewhat interested & 18 \\
\hline Not very interested & 21 \\
\hline Not at all interested & 34 \\
\hline Or do you already use telehealth to connect with a healthcare provider & 14 \\
\hline Don't know/refused (do not read) & 1 \\
\hline
\end{tabular}

Q36. (Among those who said they are extremely, very, or somewhat interested in telehealth services) What would you want to use telehealth services for? (Randomize) (Select all that apply)

\begin{tabular}{|l|c|}
\hline & $\begin{array}{c}\text { Total } \\
\text { (\%) }\end{array}$ \\
\hline & 450 \\
\hline To renew prescriptions & 72 \\
\hline For a routine visit to the doctor & 67 \\
\hline For diagnosing an illness & 50 \\
\hline To discuss a new medical issue & 62 \\
\hline To get a second opinion & 54 \\
\hline To get help in providing care to a loved one & 59 \\
\hline None of the above (do not read) (exclusive) & 3 \\
\hline Don't know/refused (do not read) & 2 \\
\hline
\end{tabular}


Q37. What are some things that might prevent you from using telehealth services? (Randomize) (Read list; select all that apply)

\begin{tabular}{|l|c|}
\hline & $\begin{array}{c}\text { Total } \\
(\%)\end{array}$ \\
\hline & 1,504 \\
\hline You don't have a computer & 18 \\
\hline You don't have access to high-speed internet service & 20 \\
\hline You don't know how to use telehealth & 31 \\
\hline You are concerned about your health information remaining confidential & 25 \\
\hline You are concerned about the possibility of medical errors & 28 \\
\hline You are not sure that your doctor's office offers telehealth services & 31 \\
\hline Your doctor's office does not offer telehealth services & 19 \\
\hline You are not sure that your insurance covers telehealth services & 30 \\
\hline The health issue can't be resolved by video or teleconference & 39 \\
\hline Something else (anchor) & 17 \\
\hline None of the above (do not read) & 12 \\
\hline Don't know/refused (do not read) & 3 \\
\hline
\end{tabular}

Q38. Have you or a family member used telehealth in the past six months?

\begin{tabular}{|l|c|}
\hline & $\begin{array}{c}\text { Total } \\
\text { (\%) }\end{array}$ \\
\hline & 1,504 \\
\hline Yes & 30 \\
\hline No & 67 \\
\hline Don't know/refused & 3 \\
\hline
\end{tabular}

\section{Demographic Characteristics}

D2. So I can make sure I have it recorded correctly, can you please state your gender?

\begin{tabular}{|l|c|}
\hline & Total (\%) \\
\hline & 1,504 \\
\hline Male & 47 \\
\hline Female & 53 \\
\hline Non-binary/other & $<1$ \\
\hline Refused & $<1$ \\
\hline
\end{tabular}




\section{AARP}

D3. Are you of Hispanic, Spanish, or Latino origin or descent?

\begin{tabular}{|l|c|}
\hline & Total (\%) \\
\hline Yes & 1,504 \\
\hline No & 3 \\
\hline Don't know/ref. & 96 \\
\hline
\end{tabular}

D4. What is your race? (Read list; accept multiple responses)

\begin{tabular}{|c|c|} 
& Total (\%) \\
\hline White or Caucasian & 1,504 \\
\hline Black or African American & 90 \\
\hline Native American or Alaskan Native & 6 \\
\hline Asian & 3 \\
\hline Native Hawaiian or other Pacific Islander & $<1$ \\
\hline Other & $<1$ \\
\hline Refused (VOL) & $<1$ \\
\hline
\end{tabular}

D5. Which one of the following best describes your employment status? (Read list)

\begin{tabular}{|l|c|}
\hline & Total (\%) \\
\hline Self-employed full-time & 1,504 \\
\hline Self-employed part-time & 7 \\
\hline Employed full-time & 2 \\
\hline Employed part-time & 25 \\
\hline Retired and not working at all & 6 \\
\hline Unemployed and looking for work & 48 \\
\hline Not in the labor force for other reasons & 1 \\
\hline Refused (VOL) & 11 \\
\hline
\end{tabular}

D6. What is your current marital status? (Read list)

\begin{tabular}{|c|c|} 
& Total (\%) \\
\hline Married & 1,504 \\
\hline Not married, living with your partner or significant other & 58 \\
\hline Separated & 4 \\
\hline Divorced & 1 \\
\hline Widowed & 11 \\
\hline Currently single and never married & 17 \\
\hline Refused (VOL) & 9 \\
\hline
\end{tabular}


D7. Which best describes the highest level of education you have completed or the highest degree you have received? (Read list)

\begin{tabular}{|l|c|}
\hline & Total (\%) \\
\hline 0 to $12^{\text {th }}$ grade, but with no diploma & 1,504 \\
\hline High school graduate or equivalent & 10 \\
\hline Post high school education, but with no degree & 30 \\
\hline 2-year degree/Associate's & 21 \\
\hline 4-year degree/Bachelor's & 16 \\
\hline Post graduate study, but with no degree & 12 \\
\hline Graduate or professional degree/Master's/PhD/Law & 2 \\
\hline Refused (VOL) & 9 \\
\hline
\end{tabular}

D8. What type of health insurance coverage do you have? (Read list; accept multiple responses)

\begin{tabular}{|l|c|}
\hline & Total (\%) \\
\hline & 1,504 \\
\hline Through your current employer & 20 \\
\hline Through your spouse's current employer & 9 \\
\hline Retiree benefit from your former employer & 12 \\
\hline Retiree benefit from your spouse's former employer & 5 \\
\hline Individually purchased health insurance & 19 \\
\hline Medicaid & 12 \\
\hline Medicare & 45 \\
\hline Veterans' Administration / Military & 8 \\
\hline Other & 7 \\
\hline You do not have health insurance (do not read) & 5 \\
\hline Refused (do not read) & 2 \\
\hline
\end{tabular}

D9. Are you or your spouse currently a member of A-A-R-P?

\begin{tabular}{|l|c|}
\hline & Total (\%) \\
\hline Yes & 1,504 \\
\hline No & 29 \\
\hline Not sure & 69 \\
\hline Refused & 2 \\
\hline
\end{tabular}


D10. Did you ever serve on active duty for the United States Armed Forces?

\begin{tabular}{|l|c|}
\hline & Total (\%) \\
\hline Yes & 1,504 \\
\hline No & 14 \\
\hline
\end{tabular}

D11a. Not including yourself, how many adults age 18 and older live in your household? (CODED)

\begin{tabular}{|l|c|}
\hline & Total (\%) \\
\hline & 1,504 \\
\hline None & 27 \\
\hline One & 48 \\
\hline Two & 16 \\
\hline Three or more & 7 \\
\hline Refused & 1 \\
\hline
\end{tabular}

D11b. And how many children under age 18 live in your household? (CODED)

\begin{tabular}{|l|c|}
\hline & Total (\%) \\
\hline & 1,504 \\
\hline None & 81 \\
\hline One & 8 \\
\hline Two & 7 \\
\hline Three or more & 3 \\
\hline Refused & 1 \\
\hline
\end{tabular}

D12. Finally, just for statistical purposes, please tell me is your current annual HOUSEHOLD income before taxes? (Read list)

\begin{tabular}{|l|c|}
\hline & Total (\%) \\
\hline & 1,504 \\
\hline Less than $\$ 30,000$ & 30 \\
\hline$\$ 30,000$ to $\$ 49,999$ & 17 \\
\hline$\$ 50,000$ to $\$ 74,999$ & 16 \\
\hline$\$ 75,000$ to $\$ 149,999$ & 19 \\
\hline$\$ 150,000$ or more & 7 \\
\hline Refused $(\mathrm{VOL})$ & 11 \\
\hline
\end{tabular}

